\title{
SAÚDE E TRABALHO DE MULHERES PROFISSIONAIS DE ENFERMAGEM EM UM HOSPITAL PÚBLICO DE SALVADOR, BAHIA*
}

\author{
Estela Maria Leão de Aquino** \\ Maria Jenny Silva Araujo*** \\ Greice Maria de Souza Menezes**** \\ Lilian de Fátima Barbosa Marinho+
}

\begin{abstract}
RESUMO: O trabalho de Enfermagem, desenvolvido essencialmente por mulheres, envolve numerosos fatores de risco para a saúde. Apesar disso, no Brasil, os estudos sobre a saúde desse grupo ocupacional praticamente inexistem. Este estudo transversal pretendeu diagnosticar as condições de saúde e trabalho de enfermeiras e auxiliares de enfermagem em um grande hospital público. Foram realizadas entrevistas e medidas de peso, altura e pressão arterial no próprio local de trabalho, em 497 trabalhadoras. Especialmente nas auxiliares, foram encontradas altas prevalências de problemas agudos (45\%), e crônicos como dores de coluna (71\%), varizes $(57,5 \%)$, hipertensão arterial $(24,9 \%)$, transtomos mentais $(36,7 \%)$, obesidade (16\%), problemas digestivos $(34,5 \%)$ e respiratórios $(31 \%)$. Ambos os grupos apresentam excessivas jornadas de trablho profissional. Contudo, as auxiliares são duplamente penalizadas pelo acúmulo de trabalho doméstico, o que contribui para suas piores condições de saúde.
\end{abstract}

ABSTRACT: Nursing work is developed mainly by women and includes many risk factors for several diseases. Nevertheless, in Brazil, the studies about health conditions of the occupational group are almost inexistent. This cross-sectional study was carried out in order to investigate the health and work conditions of R.N. and auxiliary nurses of a public hospital. The data collection included interviews and measures of weight, height and blood pressure, in 495 employed nurses and auxiliary nurses. Specially among the auxiliary nurses, the results revealed very high prevalences of acute (45\%) and chronic conditions, such as backache $(71 \%)$, varices $(57,5 \%)$, arterial hypertension $(24,9 \%)$, mental disorders $(36,7 \%)$, obesity $(16 \%)$, digestive $(34,5 \%)$ and respiratory problems $(31 \%)$. Both groups have too long a professional workday. However, the auxiliary nurses are overloaded by homework, that contribute to worsen their health conditions.

\section{INTRODUÇÃO}

A atividade de enfermagem tem sido desenvolvida, tradicionalmente, por mulheres. Antes, executada nos lares, como encargo doméstico e, depois, nos hospitais medievais, em nome da caridade cristã, a enfermagem se estruturou como profissão, dentro dos marcos do capitalismo, mantendo essa distinção

Trabalho apresentado no $45^{\circ}$ Congresso Brasileiro de Enfermagem - Recife - PE, 28 de novembro a 3 de dezembro de 1993

** Médica. Mestre em Medicina Social (IMS/UERJ. Doutoranda em Saúde Pública (DMP/UFBA). Professora assistente (IMS/UERJ) e pesquisadora do Núcleo de Estudos Mulher e Saúide (MUSA/UFBA).

*** Enfermeira, Mestre em Saude Comunitária (UFBA). Professora Adjunta da Escola de Enfermagem (UFBA). Coordenadora da Comissão de Publicações e Divulgação (ABEN-Nacional)

**** Médica, mestranda em Saúde Comunitíria (UFBA). Pesquisadora (MUSA/UFBA)

+ Enfermeira, mestranda em Saúde Comunitária (IFBA). Pesquisadora (MUSA/UFBA). 
de gênero, onde a arte de curar tornou-se monopólio de elites masculinas, separada dos cuidados diretos aos pacientes, exercidos por mulheres, de modo subordinado aos primeiros. ${ }^{(9)}$

Ao institucionalizar-se em meados do século XIX, outra característica marcante da nova profissão foi a reprodução de uma divisão social do trabalho, pretensamente através de uma divisão técnica, onde as atividades de ensino e de supervisão eram exercidas pelas ladies nurses, provenientes de famílias aristocráticas, e as atividades de cuidado direto ao paciente executadas pelas nurses, de nível socio-econômico inf erior. ${ }^{(3)}$ Essa divisão permanece, atualmente, pela separação das funções "mais intelectuais" - a cargo das enfermeiras - das atividades manuais, realizadas pelas auxiliares. ${ }^{(19)}$

A profissão de enfermagem esteve sempre marcada por conteúdos fortemente ideológicos, onde as especificidades de gênero são naturalizadas, atribuindo-se a predominância feminina a uma aptidão "inata" das mulheres para cuidar dos outros, a qual supostamente faltaria aos homens. ${ }^{(34)}$ Por outro lado, encontra-se perneada pelo discurso religioso, onde são enf atizados o devotamento, o idealismo, 0 altruísmo e o desprendimento material. ${ }^{(15)}$

Estudos nacionais ${ }^{(3,15)}$ apontam para a persistência de uma visão idealizada da profissão pelas enfermeiras, que se contrapõe à dura realidade de um trabalho mal remunerado, com excessiva carga de atividades. A análise das condições de trabalho de enfermagem revestem-no de características específicas, pois além de se relacionar com a doença e com a morte, é parte de um sistema que assegura a continuidade da produção e determina a quebra da continuidade no trabalho realizado individualmente, o que define o trabalho por turnos. Estudos realizados em outras categorias profissionais evidenciam que as jornadas rotativas causam alterações do sono, distúrbios nervosos e digestivos, além de desorganizarem a vida familiar e social das trabalhadoras. No caso da enfermagem, a exposição a fatores de risco mecânicos e ambientais específicos, é ainda agravada pelos recursos materiais insuficientes e inadequados, que ocasionam condições inseguras no trabalho. ${ }^{(4.5)}$

Apesar disso, os estudos sobre condições de saúde desse grupo ocupacional, ainda que razoavel mente freqüentes em outros países $(6,11,17,21,23,24,29,30,31$. 32), no Brasil, são raros e inespecíficos. Os existentes. em geral, referem-se aos riscos potenciais de insalubridade ou ao absenteísmo por motivos de saúde do pessoal que trabalha em hospitais. $(25,26,33,35)$
Apenas na década de 80 , a enf ermagem brasileira começou a discutir sua prática como trabalho, acompanhando o processo de discussão da crise do setor saúde que evoluiu a partir da década de $70 .(3,4,5,15,19)$ Esses primeiros estudos têm em comum a ênfase em caracterizar a prática de enfermagem como trabalho e conferir-lhe, dessa forma, visibilidade social. A constatação da carência de investigações sobre as condições de trabalho têm merecido destaque em congressos e fóruns da enfermagem, onde se apontou a urgência na realização de estudos que dêem conta de suprir essa lacuna.

É provável, portanto, que dessa aparente "invisibilidade" do trabalho de enfermagem, decorra o fato de que seus efeitos sobre a saúde apenas comecem a ser investigados.

O estudo sobre as condições de saúde desse grupo profissional, deve levar em consideração a complexidade das relações entre saúde e trabalho, que extrapolam a visão tradicional da saúde ocupacional, restrita ao trabalho industrial e à uma visão limitada ao ambiente de trabalho.

Do ponto de vista estrito, os agravos à saúde relacionados ao trabalho são classificados $\mathrm{em}$ dois grupos: no primeiro, incluem-se aqueles que traduzem nuptura abrupta do equilíbrio entre as condições e o ambiente de trabalho e a saúde do trabalhador, como os acidentes do trabalho e as intoxicações agudas de origem profissional. O segundo grupo inclui agravos de caráter crônico: a doença profissional típica, definida como aquela inerente ou peculiar a determinado ramo de atividade e constante da relação organizada pelo Ministério da Previdência e Assistência Social. ${ }^{(1)}$

Um outro grupo é constituído pelas "doenças relacionadas com o trabalho", definidas pela Organização Mundial de Saúde como "agravos outros que, $\mathrm{em}$ adição a doenças profissionais legalmente desconhecidas, ocorrem $\mathrm{em}$ trabalhadores quando o ambiente ou as condições contribuem significativamente para a ocorrência de doenças, porém em graus variados de magnitude". (1) Neste grupo, o Comitê de Especialistas da OMS inclui:

- distúrbios comportamentais c doenças psicossomáticas;

- hipertensão arterial:

- doença isquêmica do coração:

- doenças respiratórias crônicas não específicas (bronquite crônica, enfisema, asma brônquica); 
- doenças do aparelho locomotor (lombalgias, artralgia de ombro e pescoço, etc);

- câncer,

- atopia (dermatites, rinite, asma brônquica, etc).

Pode-se, ainda, mencionar grupos de problemas atribuídos à organização do trabalho no modo de produção capitalista, discutidos por autores como $\operatorname{DEJOURS}^{(8)}$ e LAURELL \& NORIEGA ${ }^{(14)}$, como o envelhecimento precoce, a síndrome da fadiga patológica, os distúrbios do sono e da sexualidade, o estresse crônico.

No presente estudo, de caráter exploratório, pretendeu-se conhecer as condições de trabalho e de saúde de profissionais de enfermagem, de modo a permitir o levantamento de hipóteses para futuras análises.

Entretanto, eleger como objeto de estudo um grupo profissional, constituído quase exclusivamente por mulheres, implica na necessidade de contemplar as especificidades do trabalho feminino, em especial, no que diz respeito à incorporação do trabalho doméstico, já que sua inserção profissional não as libera das funções tradicionais de cuidado da casa e dos filhos. Assim, além de características gerais da população estudada e de aspectos relativos ao trabalho profissional, foram levantadas informações sobre o trabalho que exercem na esfera doméstica, seja quanto à extensão e à natureza das atividades. Os resultados aqui apresentados são os primeiros obtidos, já que o trabalho de campo da investigação foi recentemente encerrado e o banco de dados encontra-se $\mathrm{cm}$ fase de constituição. Entretanto, por entender a relevância do presente evento para os desdobramentos decorrentes do mais amplo uso dos resultados dessa pesquisa, foram priorizados alguns dados para apresentação. de modo a permitir um diagnóstico preliminar da situação de trabalho e saúde desse grupo.

\section{METODOLOGIA}

Foi desenvolvido um estudo de caso $\mathrm{em}$ um dos maiores hospitais públicos de Salvador-Ba*. O cstudo, de corte transversal, envolveu a entrevista de todas as profissionais de enfermagem definidas como elegíveis, através de questionário de perguntas fecha- das, além de medidas de pressão arterial sanguínea (PAS), peso e altura. Foram consideradas elegíveis todas as profissionais de enfermagem do quadro permanente da instituição, lotadas no hospital escolhido, sendo excluídas as afastadas por motivos pessoais, em licença-prêmio, à disposição de outros órgãos ou deslocadas de função. Aquelas afastadas para tratamento de saúde, licença maternidade ou férias foram consideradas elegíveis, sendo entrevistadas no retorno ou, excepcionalmente, no domicílio.

A medida de PAS envolveu duas tomadas na mesma ocasião (no início e ao final da entrevista), sempre na posição sentada, no braço direito à altura do coração. Foram considerados casos de hipertensão arterial aquelas mulheres que apresentaram, ao exame, PAS maior ou igual a $160 / 95 \mathrm{mmHg}$.

A leitura do peso foi feita com auxílio de balança de mola portátil, elétrica, com calibragem automática e alta precisão. A altura foi aferida por estadiômetro indeformável, com a mulher em pé, ereta e com os pés descalços. Foram classificadas como casos de obesidade aquelas que apresentaram Índice de Massa Corpórea $\left(\mathrm{IMC}=\right.$ peso/altura $\left.{ }^{2}\right)$ igual ou maior que 30 . Considerou-se como sobrepeso, aquelas cujo IMC era inf erior a 30 e igual ou maior que 25 , sendo normais os valores inferiores a essa faixa.

Oquestionário f oi composto dos seguintes blocos de variáveis: características sócio-demográficas; trabalho profissional e doméstico; saúde. Na parte relativa à saúde, foram investigados problemas agudos ou agudização de problemas crônicos ocorridos nos últimos quinze dias anteriores ao momento da entrevista; problemas crônicos com e sem diagnóstico clínico anterior (pressão alta e outros problemas cardio-vasculares; diabetes; problemas digestivos e renais; alergias: varizes e dor na coluna; problemas mentais, tipo ansiedade e depressão). Para investigar os problemas mentais, utilizou-se o Self Report Questionnaire SRQ-2() - um instrumento auto-aplicado, de escala bimodal (sim/não), já testado no Brasil e aplicado anteriormente por PITT A, (25) em população de trabalhadores de saúde em um hospital geral de São Paulo.

As entrevistas $\mathrm{c}$ medidas objetivas foram ef etuadas por pessoas do sexo feminino, para facilitar a interação com as entrevistadas. Foram realizadas 495 cntrevistas $\mathrm{cm}$ quatro meses de trabalho de campo, * Por razões éticas, principalmente para evitar a estigmatização da população estudada, não serí identificado o hospital onde se
efetuou a investigação. 
Gráfico 1 - Distribuição por Idade de Enfermeiras e Auxiliares

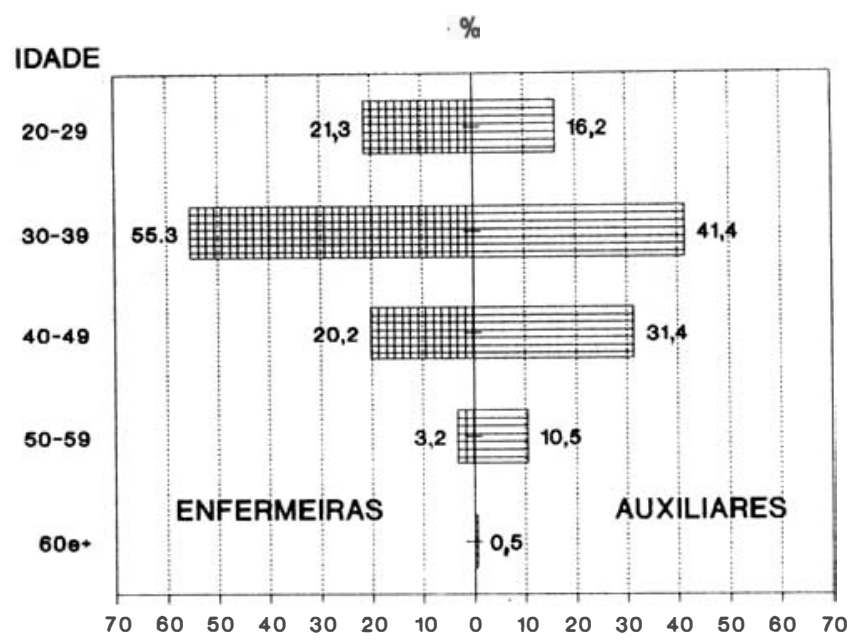

Gráfico 2 - Distribuição de Enfermeiras e Auxiliares Segundo Cor da Pele*
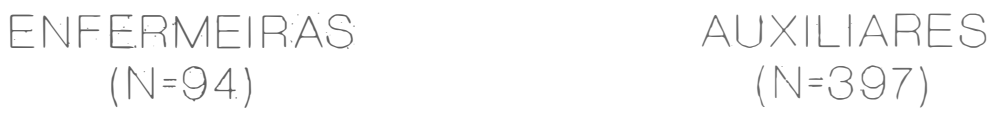

$(N=397)$

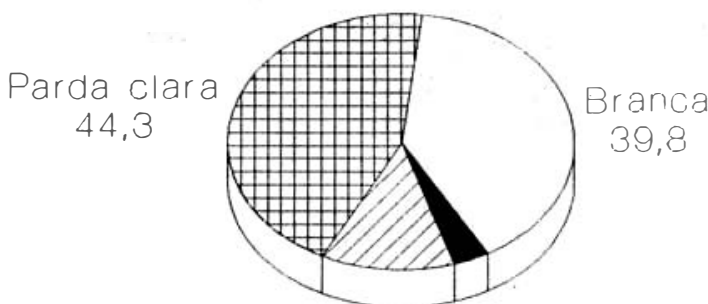

Parda escura 12.5
Negra

3,4

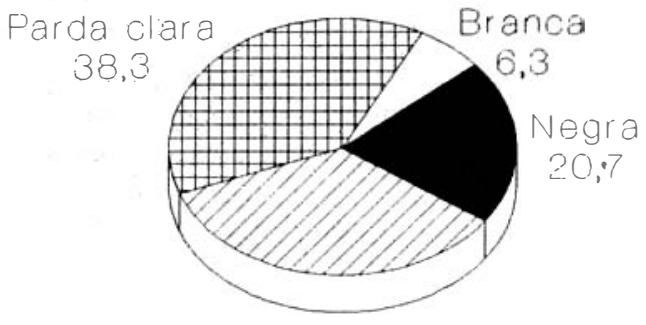

Parda escura 34,6

Gráfico 3 - Distribuição por Situação Conjugal Segundo Grupo Ocupacional

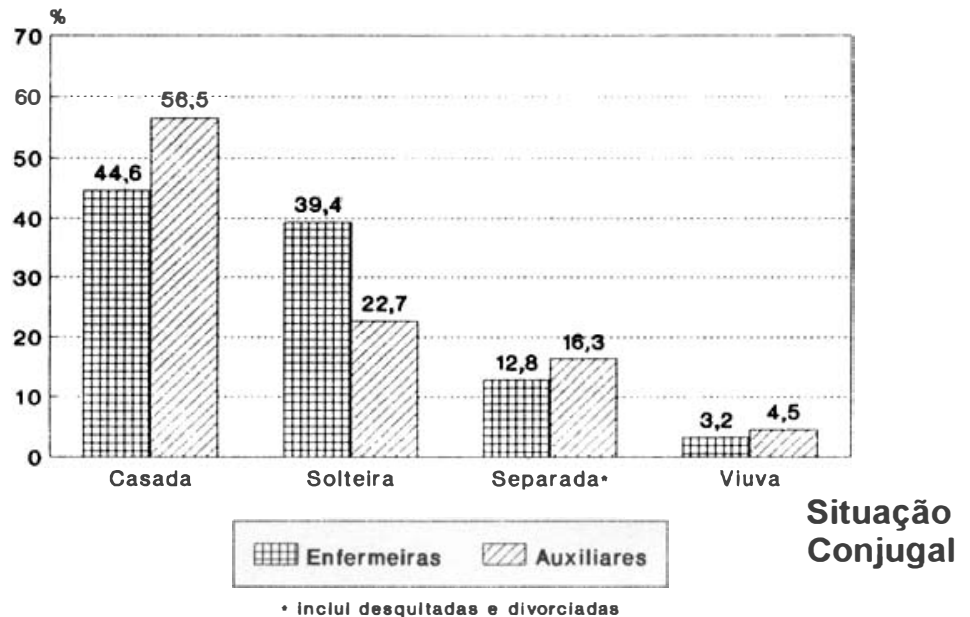




\section{Gráfico 4 - Distribuição por Chefe de Família Segundo Grupo Ocupacional}

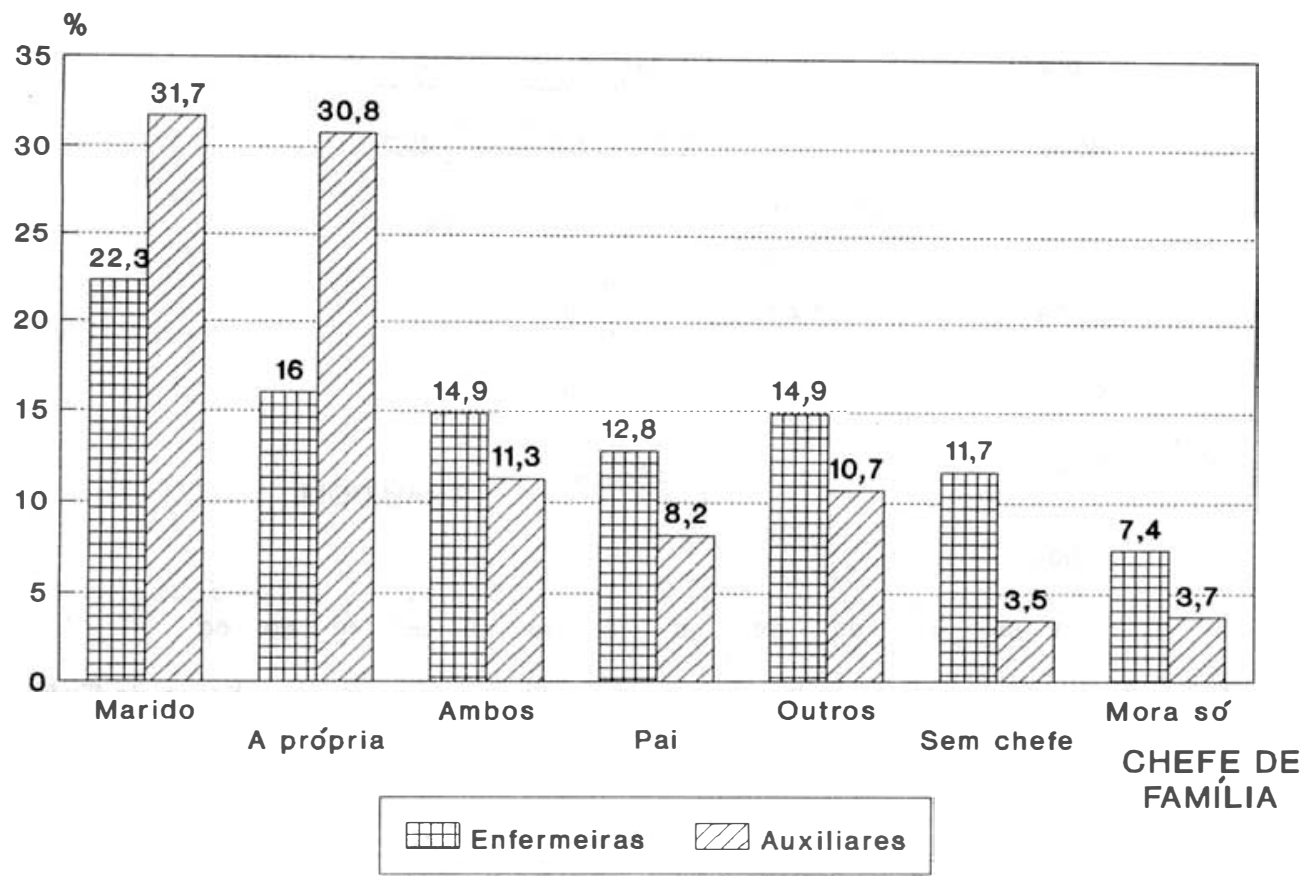

onde foram adotados procedimentos para um rigoroso controle de qualidade. Obteve-se um alto grau de colaboração, principalmente considerando-se que as entrevistas foram realizadas em ambiente de trabalho. sendo a participação de $95 \%$ do total de elegíveis, em ambos os gnupos profissionais.

O processamento de dados foi feito em microcomputador PC-AT, sendo esses primeiros resultados obtidos pelo uso do pacote Epi-Info (CDC/USA).

\section{RESULTADOS}

\subsection{Características sócio-demográficas}

A população estudada é composta de $19 \%$ de enfermeiras e $81 \%$ auxiliares de enfermagem, as quais incluem antigas atendentes quase todas reclassificadas após treinamento na própria instituição.

As médias de idade foram, respectivamente, de 35,1 e 38,3 anos, sendo que $55,3 \%$ das enfermeiras e $41,4 \%$ das auxiliares têm entre 30 e 39 anos (Gráfico 1).

Em relação à cor da pele (auto-referida), constata-se que mais da metade das auxiliares foram classificadas como sendo de cor parda escura e negra. com uma parcela muito restrita $(6,3 \%)$ de brancas (Gráfico 2). De modo oposto, uma proporção expressiva de enfermeiras ref cre ser de cor branca (39.8\%) ou parda clara $(44,3 \%)$, com uma proporção míni ma de negras
$(3,4 \%)$.

Entre as enfermeiras, um alto percentual $(39,4 \%)$ é constituído de mulheres solteiras, quase equivalente ao de casadas* (44,6\%). Das demais, $12,8 \%$ são separadas, desquitadas ou divorciadas e $3,2 \%$, viúvas. Entre as auxiliares (Gráfico 3), apenas 22,7\% são solteiras. Das que possuem companheiro $(56,5 \%)$, as casadas corresponderam a $38,5 \%$ do total de mulheres, enquanto $18 \%$ tem união consensual. As proporções de casamentos desfeitos (16,3\%) e de viúvas $(4,5 \%)$ foram um pouco superiores às das enfermeiras. Também em relação à experiência de maternidade foram grandes as diferenças: têm fillhos $55,3 \%$ das enfermeiras e 73,9\% das auxiliares. (Gráfico 3)

É interessante que $30,8 \%$ das auxiliares são chefes de família contra apenas $16,0 \%$ das enfermeiras (Gráfico 4). Em contrapartida, as enfermeiras expressam relações de coabitação mais igualitárias com proporções mais altas de ausência de chefe $(11,7 \%)$, ou de casais onde ambos são chefes (14,9\%) (Gráfico 4).

\subsection{Trabalho}

As trabalhadoras de enfermagem estudadas têm dois padrões claros e distintos quanto à qualificação profissional. As auxiliares de enfermagem, que co- 
Gráfico 5 - Duração Semanal do Trabalho Doméstico e Profissional de Enfermeiras

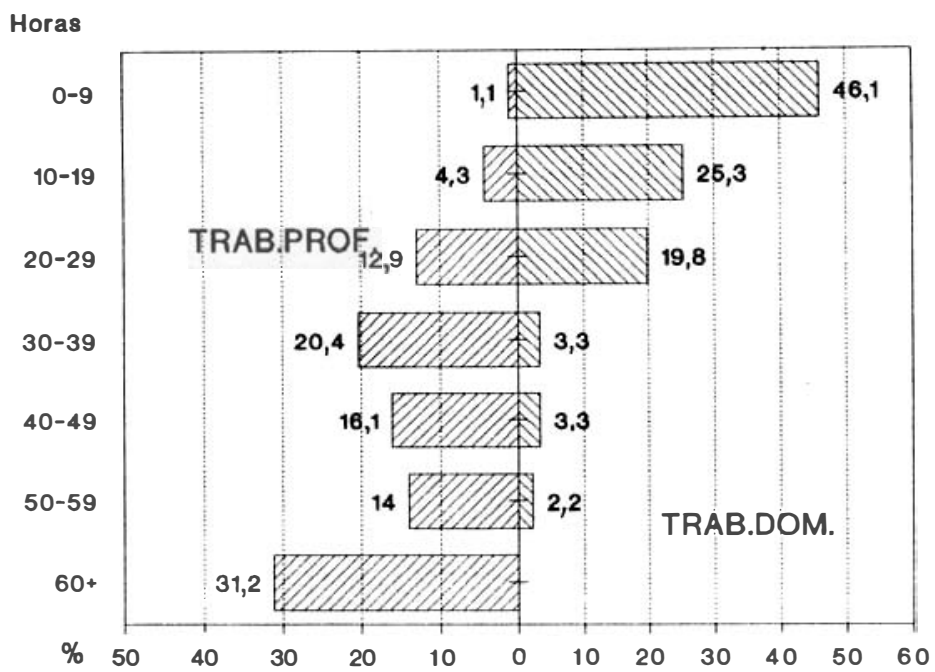

Gráfico 6 - Duração Semanal do Trabalho Doméstico e Profissional de Auxiliares de Enfermagem

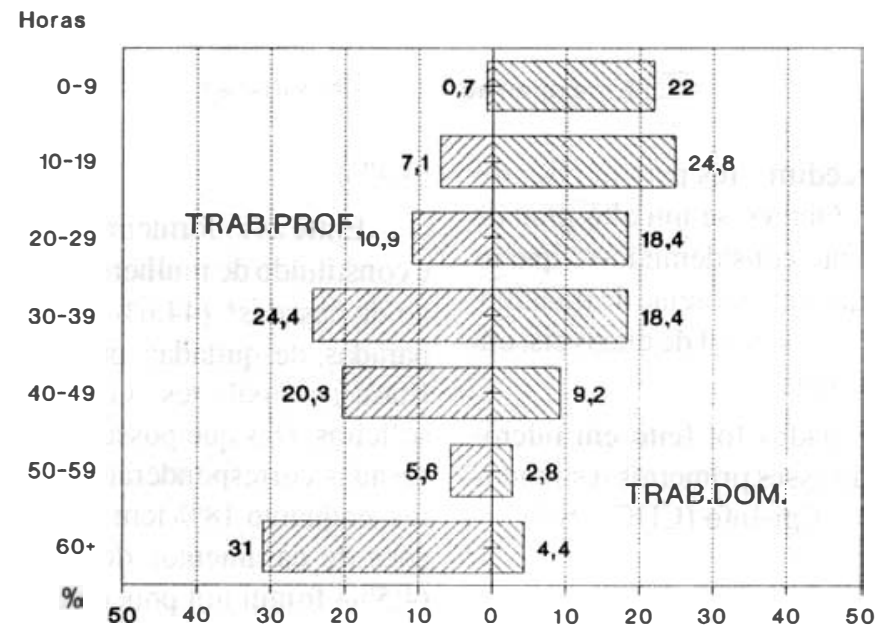

Gráfico 7 - Distribuição por Tumo de Trabalho Segundo Grupo Ocupacional

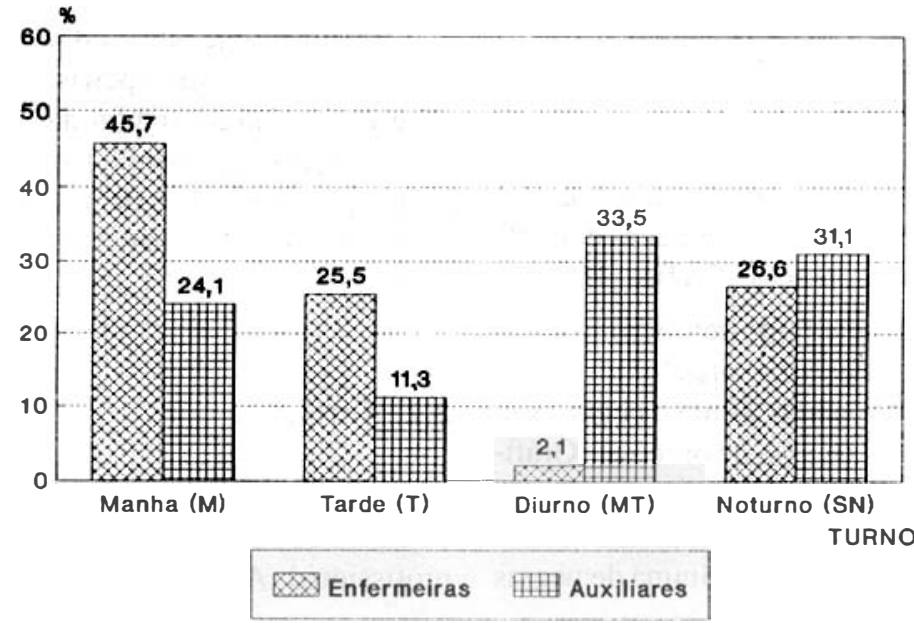




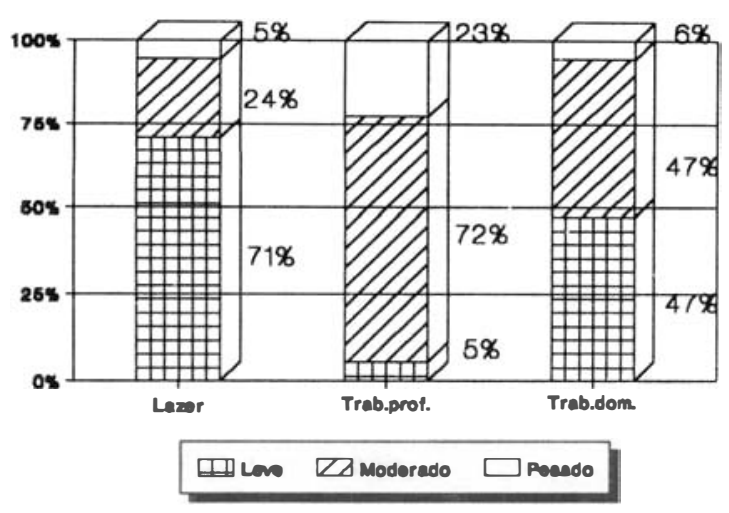

ENFERMEIRAS

meçaram a trabalhar cedo, geralmente como atendentes, apresentam uma trajetória de progressiva qualificação, como é possível visualizar-se no Quadro 1. Uma parcela minoritária $(7,5 \%)$ permanece ainda como atendente, tendo a grande maioria $(87,7 \%)$ nível máximo de qualificação de auxiliar de enfermagem. Uma pequena proporção $(4,8 \%)$ tem curso técnico de enfermagem, embora permaneçam enquadradas como auxiliares.

Quadro 1 - Cursos de Enfermagem e Nivel Máximo de Qualificação de Auxiliares

\begin{tabular}{lr}
\hline NÍVEL MÁXIMO / OUTROS CURSOS & $\%$ \\
\hline Atendente de Enfermagem & $\underline{7,5}$ \\
Atendente & 4,8 \\
Atendente + outros cursos & 2,7 \\
& \\
Auxiliar de Enfermagem & $\underline{87,7}$ \\
Atendente + auxiliar & 50,4 \\
Atendente + auxiliar + outros & 5,5 \\
Auxiliar & 27,0 \\
Auxiliar + outros & 4,8 \\
& \\
Técnico de Enfermagem & 4,8 \\
Auxiliar + técnico & 1,7 \\
Auxiliar + técnico + outros & 0,3 \\
Técnico & 1,0 \\
Atendente + Auxiliar + Técnico & 1,8 \\
\hline
\end{tabular}

As enfermeiras, em sua maioria, têm apenas o curso de graduação, e apenas uma entre as noventa e quatro entrevistadas tinha pós-graduação senso estrito, com curso de mestrado. Duas delas estavam, respectivamente, fazendo curso de medicina e ciências biológicas.

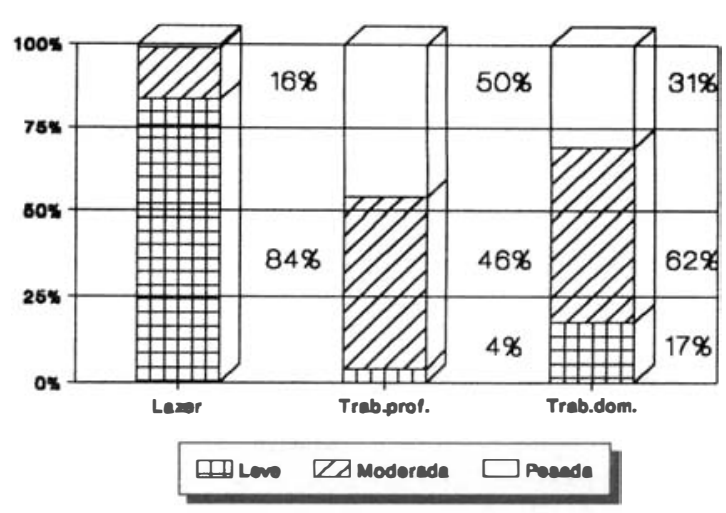

AUXILIARES

Ambos os grupos ocupacionais caracterizam-se por uma carga de trabalho profissional bastante expressiva (Gráficos 5 e 6). Isso se expressa tanto em relação à existência de mais de uma atividade regular remunerada $(59,6 \%$ das enfermeiras e $53,0 \%$ das auxiliares), quanto na carga horária semanal (com médias de 46,6 horas para as enfermeiras e 45,7 horas para as auxiliares).

Contudo, as diferenças são marcantes quanto ao regime de turno e o tipo de atividade que desenvolvem em sua segunda jomada, demonstrando a relação entre o tipo de inserção econômica e a qualificação profissional. Assim, as enfermeiras são mais numerosas em turnos de seis horas $(71,2 \%)$, sendo sua maior presença no hospital estudado pela manhã (45.7\%). A opção pelo esquema de plantão se dá quase exclusivamente pelo turno noturno $(26,6 \%)$. Em sua quase totalidade (92,9\%), as enfermeiras têm como outra atividade um emprego hospitalar ou na área de saúde, provavelmente em uma segunda jornada diária de seis horas, no turno da tarde. (Gráfico 7)

As auxiliares estão inseridas em plantões de doze horas, sejam diumos $(33,5 \%)$ ou notumos $(31,1 \%)$. Além desse emprego, muitas possuem atividades variadas, parte das quais informais, como a costura e o artesanato, o ensino particular, a venda de confecções, bijuterias e produtos de beleza, atividades de manicure e cabeleireira.

Também em relação ao esf orço físico no trabalho profissional, acentuam-se as diferenças. Enquanto $71,3 \%$ das enfermeiras submetem-se a esforço físico moderado (caminham muito, mas não carregam peso), a quase totalidade das auxiliares tem esforço físico pesado $(46,4 \%)$, ou moderado $(49,6 \%)$ (Gráfico 8$)$. 
Gráfico 9 - Sobrecarga Doméstica* Segundo Tipo de Atividade e Grupo Ocupacional

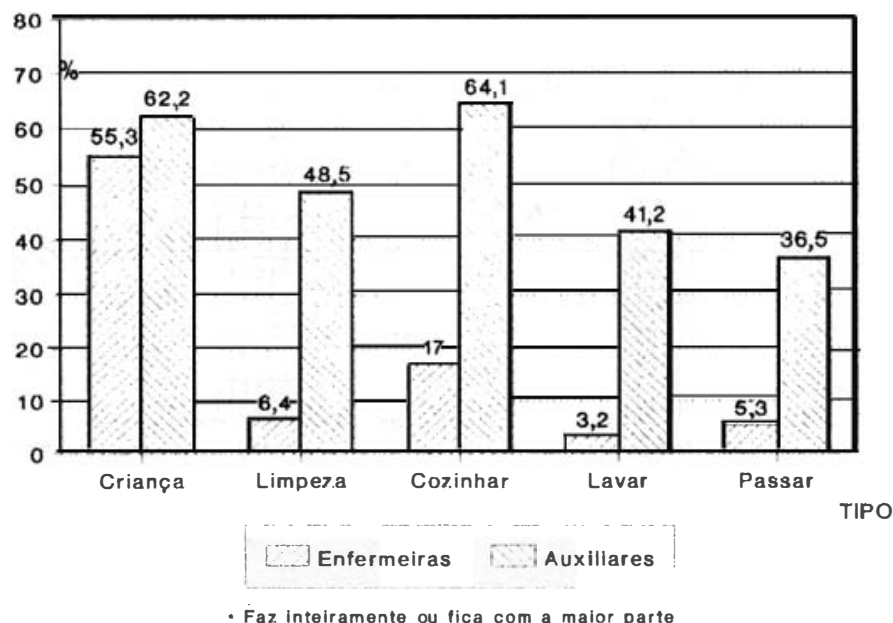

Gráfico 10 - Prevalência de Sobrepeso e Obesidade em Enfermeiras e Auxiliares

\section{ENFERMEIRAS \\ $(N=94)$}

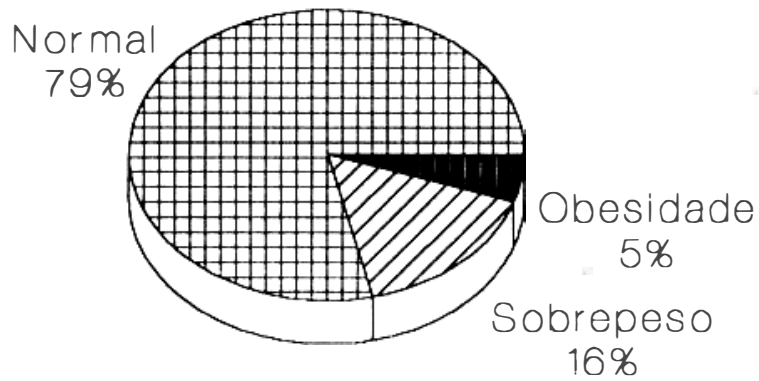

AUXILIARES

$(N=400)$

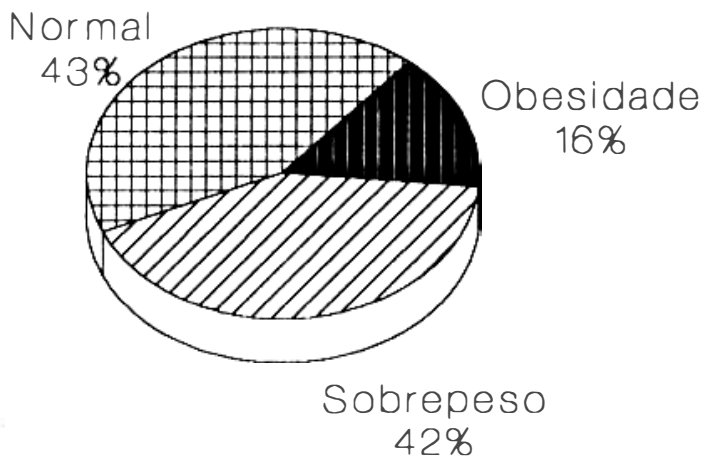

Gráfico 11 - Prevalência de Hipertensão Arterial em Enfermeiras e Auxiliares

ENFERMEIRAS

$(N=94)$

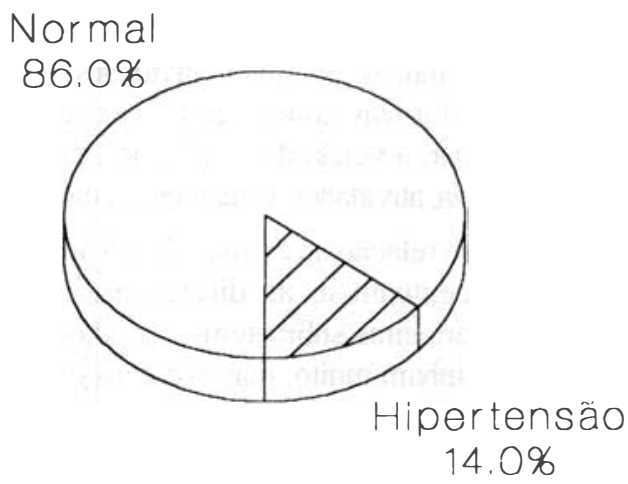

AUXILIARES

$(N=400)$

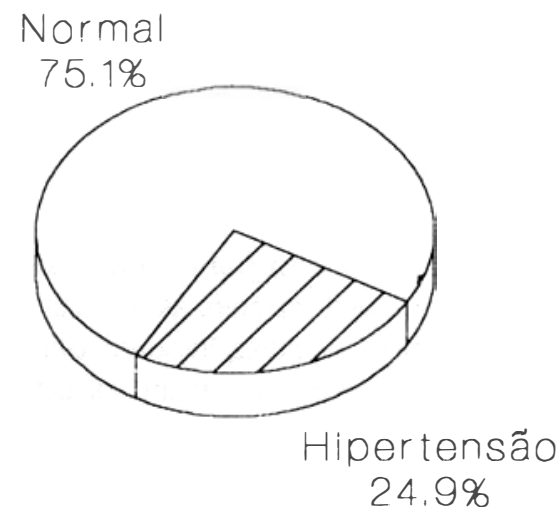



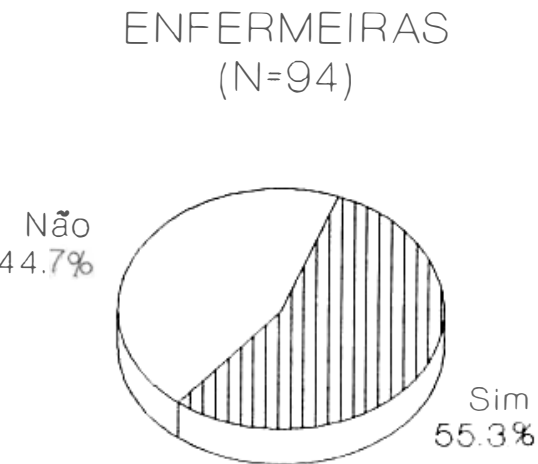
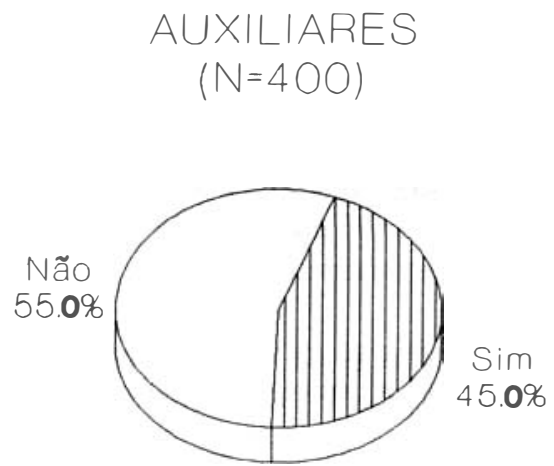

Gráfico 13 - Problemas Mentais Menores* Segundo Grupo Ocupacional
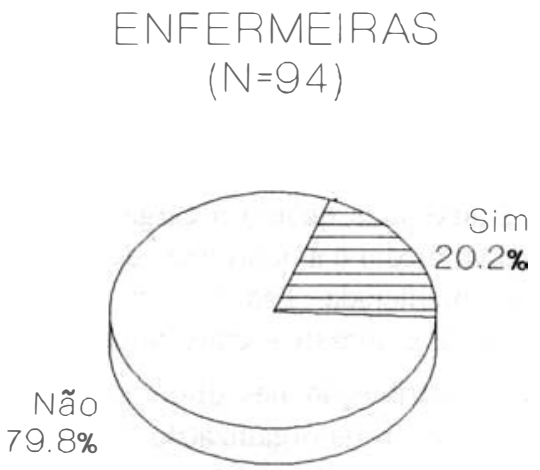

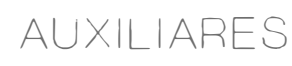

$(N=400)$

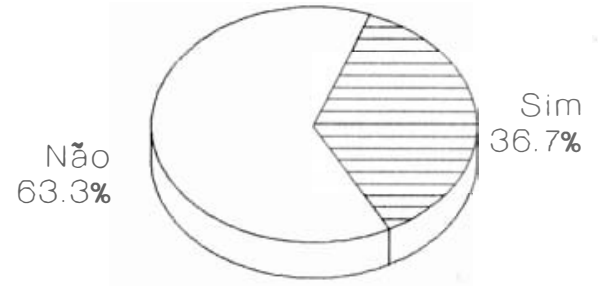

*tipo ansiedade e depressåo

Quando analisado o trabalho doméstico, as distinções são ainda mais expressivas (Gráfico 5 e 6). As enfermeiras têm, em média, 13,9 horas semanais de atividades de cuidado da casa e dos filhos, enquanto as auxiliares têm, praticamente, o dobro, com 24,2 horas. Também o esforço desenvolvido nesse tipo de atividade é para as enfermeiras leve $(42,6 \%)$ ou moderado $(47,9 \%)$, enquanto para as auxiliares é, em sua maioria, moderado $(51,6 \%)$ ou pesado $(30,9 \%)$ (Gráfico 8).

Se analisadas as atividades desenvolvidas no trabalho doméstico, fica evidente que, ainda que o cuidado das crianças seja uma incumbência das mulheres de ambos os grupos, as diferenças quanto às demais são bastante marcantes (Gráfico 9). As auxiliares, pelo fato de terem uma atividade profissional com grande carga horária semanal, não são poupadas em absoluto das tarefas de casa. Quando não estão no hospital, grande parte delas realiza inteiramente, ou a maior parte, das atividades de limpeza (48,5\%), cozinha $(64,1 \%)$, além de lavar e passar $(41,2 \%$ e $36,5 \%)$. As enfermeiras contam com outras formas de apoio, especialmente as empregadas domésticas, que as li- beram em sua maioria dessas tarefas. (Gráfico 9).

\subsection{Saúde}

As marcantes diferenças observadas vão se refletir no padrão de adoecimento de ambos os grupos, com piores condições de saúde para as auxiliares de enfermagem.

Esse grupo apresentou altíssimas prevalências de obesidade (16\%) e sobrepeso (42\%), se comparadas àquelas identificadas entre as enfermeiras (respectivamente, $5 \%$ e $16 \%$ ) (Gráfico 10 ).

Em ambos os grupos, mas especialmente entre as auxiliares, a prevalência de hipertensão arterial foi muito alta (Gráfico 11), principalmente considerando-se as médias de idade.

A morbidade aguda referida (Gráfico 12) foi, também, muito elevada com prevalência de problemas de saúde nos últimos quinze dias de $55,3 \%$ entre as enfermeiras e de $45,0 \%$ entre as auxiliares.

A referência de problemas de saúde crônicos foi, em ambos os grupos, especialmente freqüente quanto 
à ocorrência de dor na coluna, varizes, pressão alta, problemas digestivos e alérgicos, respiratórios e de pele (Quadro 2). Ainda que, a maior parte desses tenha ocorrido de modo tão freqüente entre enfermeiras e auxiliares, ressalte-se a dor na coluna, as varizes e a pressão alta que foram mais prevalentes nos relatos das auxiliares de enfermagem.

Quadro 2 - Prevalência de Problemas Crônicos de Saúde Referidos por Enfermeiras e Auxiliares

\begin{tabular}{l|r|r}
\hline \multirow{2}{*}{ PROBLEMA } & \multicolumn{2}{|c}{ PREVALÉNCIA (\%) } \\
\cline { 2 - 3 } & Enfermeiras & Auxiliares \\
\hline Pressåo Alta & 38,3 & 48,9 \\
Infarto do coraçăo* & - & 1,0 \\
Insuficiência cardíaca* & -1 & 1,3 \\
Angina* & 2,1 & 1,8 \\
Derrame cerebral* & - & 0,8 \\
Outras doenças do coraçăo* & 5,3 & 5,0 \\
Diabetes & 1,1 & 1,0 \\
Úlcera digestiva* & 1,1 & 4,3 \\
Doença renal* & 5,3 & 2,8 \\
Varizes & 45,7 & 57,5 \\
Alergia de pele & 20,2 & 25,5 \\
Dor na coluna & 50,0 & 71,0 \\
Rinite, sinusite, & & \\
bronquite ou asma & 36,2 & 31,0 \\
Problemas digestivos & 36,2 & 34,5 \\
\hline \multicolumn{2}{c}{ "Com diagnóstico médico } & \multicolumn{2}{|c}{}
\end{tabular}

Mais ainda, os problemas mentais tipo ansiedade e depressão, foram quase duas vezes mais frequientes nas auxiliares (Gráfico 13).

\section{DISCUSSÃO}

A carga horária contratual da população estudada é de trinta horas semanais. Contudo, as cargas horárias médias de trabalho profissional, de um modo geral, resultantes da existência de mais de uma atividade remunerada, são superiores inclusive ao limite de quarenta e quatro horas estabelecido na Constituição brasileira para os trabalhadores em geral.

Por outro lado, a análise da jomada semanal não pode deixar de considerar as horas dispendidas em atividades domésticas, o que eleva a média laboral para 60,5 horas nas enfermeiras e para 70,6 horas nas auxiliares de enfermagem.

Acrescente-se, que para uma expressiva proporção das auxiliares de enfermagem, tanto a atividade profissional quanto a doméstica são consideradas pesadas $(46,4 \%$ e $30,9 \%$, respectivamente), o que pode contribuir para o aparecimento de fadiga crônica, desgaste físico, além de patologias específicas.

O limite máximo da jornada de trabalho das trabalhadoras de enfermagem de 30 horas semanais é reivindicação constante da categoria, e atualmente está proposto em projetos de lei, tramitando no Congresso Nacional. Embora esta limitação signifique inegavelmente uma conquista, é necessário que se atente para o fato de que, numa profissão mal remunerada e exercida predominantemente por mulheres, poderá significar apenas a possibilidade de conciliar mais facilmente os vários empregos e o trabalho doméstico, caso não seja acompanhada de outras conquistas como ganhos salariais reais, existência de equipamentos coletivos e maior participação dos homens nas atividades domésticas.

Observa-se ainda, que embora a população estudada pertença a um mesmo grupo profissional, suas relações com o trabalho são permeadas por questões de classe e de gênero.

Não existe dif erença significativa entre enfermeiras e auxiliares quanto à carga horária de trabalho semanal média e à ocorrência de mais de uma atividade remunerada. Todavia, em alguns aspectos há diferenças expressivas entre enfermeiras e auxiliares.

A distribuição nos diferentes turnos reflete as características da organização do trabalho em enfermagem, pelas quais, por exemplo, as enfermeiras realizam atividades predominantemente administrativas, que são concentradas no turno da manhã.

Una outra dif erença diz respeito a ocorrência de atividades fisicas consideradas pesadas, sejam domésticas ou profissionais, que é significativamente maior para as auxiliares de enfermagem. Isto reflete não só a divisão de trabalho existente (trabalho intelectual $\mathrm{X}$ trabalho manual), como a grande limitação das auxiliares de enfermagem de aquisição de equipamentos domésticos, bem como de contar com os serviços de empregada doméstica.

Em relação à sobrecarga doméstica, esta não só é maior para as auxiliares de enfermagem, como foi dito anteriormente, como é mais uniformemente distribuída entre as diferentes atividades (cuidar de crianças, limpeza, cozinha, lavar e passar). Diferentemente, as enfermeiras têm a sobrecarga concentrada no cuidado de crianças, que ocorre em proporção similar às auxiliares, revelando que esta atividade é fortemente associada à condição de mulher, mesmo em diferentes classes sociais.

Quanto à saúde, o grupo ocupacional estudado caracteriza-se por altas prevalências de problemas de saúde agudos e crônicos.

Os valores observados na morbidade referida 
poderiam ser explicados, tão somente, por uma percepção mais acentuada dos sintomas e uma maior propensão a valorizá-los, decorrente da própria inserção profissional. Sendo composto exclusivamente de mulheres, descritas como mais propensas que os homens a perceber e relatar seus problemas de saúde ${ }^{(22)}$, o grupo estudado, além disso, encontra-se exposto a intenso processo de medicalização. Todavia, os altos valores igualmente obtidos nas mensurações objetivas de pressão arterial sanguínea, peso e altura, sujeitas a rigoroso controle de qualidade, atestam a gravidade das condições de saúde do grupo estudado, reforçando as hipóteses de associação real da ocorrência de doenças ao trabalho que este exerce. Isso se acentua ao considerar-se o efeito de seleção de populações trabalhadoras - também chamado de " efeito do trabalhador sadio" - pelo qual pessoas sadias tenderiam a ser mais aptas a obter e a manter uma inserção profissional. Some-se a isso também, o fato de que, sendo profissionais de saúde, seria esperado que apresentassem melhores condições de prevenir, diagnosticar e tratar seus problemas, tendo como resultado um padrão mais sadio do que a população em geral.

A prevalência de hipertensão arterial é alta tanto em enfermeiras quanto em auxiliares. Mas entre essas últimas pode ser considerada alarmante, principalmente se levado em conta que essas mulheres encontram-se em idade reprodutiva, com média etária relativamente baixa.

Os resultados são, entretanto, consistentes com observações anteriores de que existiria uma associação inversa entre a ocorrência da doença e a situação sócio-econômica ${ }^{(7,36)}$. A exemplo do observado entre as auxiliares e as enfermeiras; estudo realizado em Volta Redonda ${ }^{(12)}$, com população industrial masculina, identificou maior prevalência de hipertensão arterial entre os trabalhadores não especializados, com menores salários.

A obesidade - fator de risco para aquela doença bastante bem estabelecido - revelou-se também muito freqüente entre as auxiliares, sendo consistente com a maior prevalência de hipertensão nesse grupo. É interessante ressaltar que essas mostram-se tão sedentárias quanto as enfermeiras. Todavia, sendo suas atividades físicas no trabalho - tanto profissional como doméstico - mais pesadas que as das últimas, provavelmente as diferenças no padrão de obesidade e sobrepeso devam ser explicadas por dietas inapropriadas das primeiras. Aqui, cabe notar que o trabalho por turnos principalmente o noturno, mais freqüente entre as auxiliares, tem sido descrito como associado a distúrbios alimentares, que contribuiriam para a ocorrência de problemas digestivos e nutricionais.

Outra linha de estudos tem procurado investigar o estresse ocupacional e sua relação com doenças como a hipertensão arterial e os transtornos mentais. Ainda que não haja consenso quanto aos mecanismos que determinam a elevação da pressão arterial, a relação entre o estresse ocupacional e a hipertensão arterial encontra-se bem documentada na literatura.

No Brasil, RIBEIRO e colaboradores(27), em estudo realizado no município de São Paulo, encontraram diferenças significativas entre grupos ocupacionais, com as prevalências mais altas entre trabalhadores de empresas jornalísticas e publicitárias $(21,0 \%)$, de setores de transporte públicos $(18,9 \%)$, de setores bancários e securitários $(18,6 \%)$ e de indústrias metalúrgicas $(17,3 \%)$. As diferenças foram atribuídas a fatores ligados à organização do trabalho, ao ritmo, à duração do trabalho e ao estresse inerente a algumas dessas ocupações. De fato, estudo específico em grupo de mulheres trabalhadoras de diversos setores da economia, encontrou associação positiva entre elevação de pressão arterial e o número diário e semanal de horas trabalhadas ${ }^{(28)}$.

No presente estudo, essas análises preliminares atestam jornadas de trabalho extremamente longas, com diferenças significativas entre auxiliares e enfermeiras, principalmente no trabalho doméstico.

As pressões decorrentes do trabalho não se restringem, portanto, ao âmbito profissional, mas resultam do próprio esforço de conciliação entre as atividades remuneradas e as domésticas realizadas de modo gratuito para toda a família.

Essas condições de vida e de trabalho certamente vão também se refletir na ocorrência de transtornos mentais tipo ansiedade e depressão, que foi especialmente freqüente entre as auxiliares de enfermagem.

Estudos realizados em diversas categorias profissionais, utilizando diferentes metodologias, têm evidenciado a associação entre a ocorrência de distúrbios psíquicos e aspectos como jornadas prolongadas, intensidade do ritmo de trabalho, insuficiência de pausas, conteúdo de tarefas de pouco significado ${ }^{(10)}$. Um estudo realizado com mulheres, na Região Metropolitana de Salvador, mostrou, entre outros fatores, a extensão da jornada de trabalho e o número de atividades simultâneas ao trabalho, como associados à prevalência de distúrbios mentais. ${ }^{(2)}$

A prevalência de transtornos mentais, identificada pelo SRQ-20 em diferentes países e em populações 
diversas, tem variado com o tipo de população estudada, sendo os mais baixos valores encontrados em grupos especiais de trabalhadores e os mais altos em usuários de senviços de saúde. Os estudos de base populacional geralmente apresentam valores intermediários aos dois grupos. ${ }^{(16)}$

No presente estudo, as enfermeiras apresentaram uma prevalência semelhante às encontradas em outros grupos de trabalhadores, inclusive de hospitais, que se situam em torno de $20 \%(25)$. As auxiliares, todavia, apresentaram valor bem mais elevado, aproximando-se dos identificados entre usuários de serviços de saúde, os quais constituem gnupos selecionados, pois geralmente a demanda por atenção é motivada pela percepção de problemas ${ }^{(16)}$.

As jornadas extensas de trabalho, envolvendo grande esforço físico, no âmbito profissional (para ambos os grupos) e doméstico (para as auxiliares) provavelmente vão se refletir nas altas prevalências de dor na coluna, além de intensa fadiga crônica.

As afecções do aparelho locomotor em trabalhadores têm sido descritas em diversos estudos, repre- sentando, em 1983, a terceira causa de aposentadoria por invalidez, no Brasil.(18) Entre essas afecções, a ampla maioria constitui-se de problemas de coluna. Uma de suas manifestações mais comuns, a dor lombar, costuma ter causainespecífica, estando associada a problemas ergonômicos ou traumáticos. ${ }^{(13)}$ Aparece com frequência mais elevada em trabalhadores que, como o grupo estudado, exercem atividades pesadas, de ritmo intensivo e em condições anti-ergonômicas, estando bem documentada a importância do esforço de flexão, principalmente pela posição incorreta de erguimento de pesos. ${ }^{(20)}$

Os presentes resultados apontam para a necessidade do aprofundamento da análise, no sentido de testar as hipóteses aqui indicadas. De qualquer forma, essas primeiras interpretações sugerem a conjugação de diferentes fatores, intrinsecamente relacionados à condição feminina das profissionais estudadas, que estariam determinando péssimas condições de saúde. Às questões de gênero se articulam as de classe, configurando uma situação particularmente grave para as auxiliares de enfermagem.

\section{REFERÊNCIAS BIBLIOGRÁFICAS}

1. ABRASCO. Associação Brasileira de Saúde Coletiva. Saide e Trabalho: desafio para uma politica. Rio de Janeiro: ABRASCO, 1991.

2. AGUIAR,W.M. Condições de trabalho feminino e transtornos mentais. (Dissertação de Mestrado em Saúde Comunitária) Salvador: DMP/UFBA, 1988.

3. ALMEIDA,M.C.P. \& ROCHA,J.S.Y. O saber da enfermagem e sua dimensão prática. São Paulo: Cortez, 1986 $128 \mathrm{p}$.

4. ALVES,D.B. Mercado e condições de trabalho na enfermagem. Salvador: Gráfica Central Ltda., 1987. p. 108

5. Condições de trabalho na enf enmagem - aspectos teóricos. In: $39^{\circ}$ Congresso Brasileiro de Enfermagem. 23 a 27 de novembro de 1987. Anais... Salvador: 1988. $104 \mathrm{p}$.

6. BECKER-CARUS,C. et al. Stress and situation specific coping behavior in intensive care unit nursing staff. Work and stress 3 (4): 353-8, 1989.

7. COSTA, E.A. Hipertensão arterial como problema de massa no Brasil: Caracteres epidemiológicos e fatores de risco. Ciência e Cultura; v. 35, n. 11, p. 1642-9, 1983.

8. DEJOURS,C. A loucura do trabalho: estudo de psicopatologia do trabalho. São Paulo: Oboré, 1987, 163 p.

9. EHRENREICH,B. \& ENGLISH,D. Brujas, comadronas y enfermeras. In: Enfermedad y sociedad. Medellin: s.e, 1979. p.148-71.

10. FERNANDES,S.R.P. Trabalho informatizado e disturbios psico-emocionais: estudo seccional em tres empresas de processamento de dados em Salvador, Bahia. Dissertação de Mestrado em Saúde Comunitária (DMP/UFBA), Salvador, 1992.
11. GRAY-TOFT,P. \& ANDERSON,J.G. Stress among hospital nursing staff: its causes and effects. Soc.Sci.Med. 15 A:639-47, 1981.

12. KLEIN,C.H. \& ARAÚJO,J.W.G. Fumo, bebida alcoólica, migração, instrução, ocupação, agregação familiar e pressão arterial em Volta Redonda. Rio de Janeiro. Cad.Saude Pública (RJ), v. 1, n. 2, p. 160-76, 1985.

13. KNOPLICK,J. A importância das dores na coluna na prática médica e na indústria. Rev.Bras. Saude Ocupac. v. 9, n. 36, p. 71-4, 1981

14. LAURELL,A.C. \& NORIEGA,M. Processo de produção $e$ sauide: trabalho e desgaste operário. São Paulo: Hucitec, 1989.

15. LOPES,M.J.M. O trabalho da enfermeira: nem público, nem privado, feminino, doméstico e desvalorizado. Rev. Bras. Enferm. Rio de Janeiro, v. 41, n. 3/4, p. 211-7, 1988.

16. MARI,J. Minor psychiatric morbidity in three primary medical care clinics in the city of São Paulo. Thes is (Doctor of Philosophy), University of London - Institute of Psychiatry, London, 1986.

17. MCGRATH,A. et alli. Occupational stress in nursing. Int. $J$. Nursing Studies 26(4): 343-358, 1989.

18. MEDINA,M.C.G. A aposentadoria por invalidez no Brasil. (Dissertação de Mestrado). São Paulo: USP, 1986

19. MELO,C. Divisão șocial do trabalho em enfermagem. São Paulo: Cortez, 1986. 94 p.

20. MENDES,R. O impacto dos efeitos da ocupação sobre a saúde de trabalhadores. I. Morbidade. Rev.Sauide públ. v. 22, n. 4, p. 311-26, 1988.

21. MORGAN,S.L. \& DAVIS,R. Occupational hazards: nursing service personel. Prof. Safety 34(8): 9-11, 1989 
22. NATHANSON,C.A. Sex, illness and medical care. A review of data, theory and method. Soc. Sci.Med.; 11: 13-25, 1977.

23. OWEN,B.D. The magnitude of low back problem in nursing. West.J.Nurs.Research 11(2): 234-242, 1989.

24. PARKES,K.R. Occupational stress among student nurses - 1 . A comparison of medical and surgical wards. Nursing Times 76(25): 113-6, 1980.

25. PITTA,A. Hospital, dor e morte como "oficio". $2^{\mathrm{a}}$ ed. São Paulo: Hucitec, 1991.

26. POSSO,M.B.S. As fontes potenciais de riscos fisicos e quimicos sobre os membros da equipe cirirgica. Tese de doutorado. Escola de enf ermagem da USP. São Paulo, 1988.

27. RIBEIRO,D. et al. Hypertension and economic activities in São Paulo, Brazil. Hypertension; 3 (supl.2): 233-7, 1981.

28. Hypertension among female workers in São Paulo, Brazil. Predictors and joint effects. Hypertension, Supp.V, 5 (6): V-144-8, 1983.

29. THIERNEY,D., ROMITO,P. \& MESSING,K. She ate not the bread of the idleness: exaustion is related to domestic and salaries working conditions among 539 Québec Hospital Workers. Women and Health 16(1): 21-42, 1990.
30. TOPF,M. Noise-induced occupacional stress and health in critical care nurses. Hospital topics 66(1): 30-4, 1988.

31. TRIOLO,P.K. Occupational health hazards of hospital staff nurses. Part I: Overview and psych... Am.Ass.Occup.Health Nurses J. 37(6) ; 232-7, 1989.

32. Occupational health harzards of hospital stafl nurses. Part II: Physical, Chemical. Am.Ass.Occup. Health Nurses J. 37(7): 274-9, 1989.

33. VALTORTA,A., SIDI,E. \& BIANCHI,S.C.L. Estudo do absenteismo médico num hospital geral de grande porte. Rev.Bras. Saide Ocupac., v. 13, n. 51, p. 55-61, 1985.

34. WAINERMAN,C.H. \& GELDSTEIN,R.N. Genero y vocacion entre auxiliares de enfermeria. Medicina y Sociedad; $14(1), 1991$.

35. WAKAMATSO,C.T. et alii. Riscos de insalubridade para o pessoal de hospital. Rev.Bras.Saude Ocupac., v. 14, n. 53. p. $52-60,1986$

36. WINGARD,D.L. The sex differential in morbidity, mortality and lifestyle. Ann.Rev.Public Health; 5: 433-58, 1984.

Recebido para publicação em 28.11.93 